\title{
Porcine lactoferrin-derived peptide LFP-20 modulates immune homoeostasis to defend lipopolysaccharide-triggered intestinal inflammation in mice
}

\author{
Xin Zong ${ }^{1}$, Xiaoxuan $\mathrm{CaO}^{1}$, Hong Wang ${ }^{1}$, Jing Zhao ${ }^{1}$, Zeqing $\mathrm{Lu}^{1,2}$, Fengqin Wang ${ }^{1,2}$ and Yizhen Wang ${ }^{1,2 *}$ \\ ${ }^{1}$ College of Animal Science, Zhejiang University, Hangzhou, Zhejiang Province 310058, People's Republic of China \\ ${ }^{2}$ Key Laboratory of Animal Nutrition and Feed Science in Eastern China, Ministry of Agriculture, Hangzhou, Zhejiang \\ Province 310058, People's Republic of China
}

(Submitted 5 October 2018 - Final revision received 13 February 2019 - Accepted 23 February 2019 - First published online 7 May 2019)

\section{Abstract}

The performance of immune system is vital for defending the body from pathogens, and it plays a crucial role in health homoeostasis. In a previous study, we have shown that LFP-20, a twenty-amino acid antimicrobial peptide in the $\mathrm{N}$ terminus of porcine lactoferrin, modulated inflammatory response in colitis. Here, we further investigated the effects of LFP-20 on immune homoeostasis to elucidate the mechanism of its anti-inflammation action. A lipopolysaccharide (LPS)-triggered systemic inflammatory response mice model was established. On the basis of observed mucosal lesions and apoptosis in small intestine, we found increased macrophage and neutrophil infiltration in ileum after LPS stimulation. Expectedly, LFP-20 pre-treatment attenuated the LPS-mediated immune disorders in ileum. Moreover, the flow cytometry results indicated pre-treatment with LFP-20 sustained the balance of $\mathrm{CD}^{+} \mathrm{CD}^{+} \mathrm{T}$ cells, B cells and natural killer cells in LPS-triggered immune disturbance. Simultaneously, we demonstrated LFP-20 modulated the secretion of both activated Th1-related IL-12p70, interferon- $\gamma$, TNF- $\alpha$ and Th2-related IL-4, IL-5 and IL-6. Furthermore, we found LFP-20 facilitated a balanced Th1 and Th2 response, which triggered cellular defence mechanisms and induced B cells to produce opsonising antibodies belonging to certain IgG subclasses to defend against LPS stimulation. Collectively, our study indicated pre-treatment with LFP-20 could defend against LPS-triggered systemic inflammatory response in mice via modulating immune homoeostasis.

Key words: Lactoferrin: Immune homoeostasis: Lipopolysaccharides: Inflammation

Lactoferrin (or lactotransferrin, Lf) is a glycoprotein from the transferrin family of proteins. It displayed a structural organisation shared among members of the family. That is, it is formed by a single polypeptide chain and folded into two globular lobes, which are called the N-terminal and C-terminal lobes based on their localisation ${ }^{(1)}$. It is expressed and secreted by epithelial cells in many exocrine secretions including saliva, tears and milk $^{(2,3)}$. Lf is the second most abundant protein in human milk, with a concentration varying from 1 to $7 \mathrm{gm} / \mathrm{l}$ (in colostrum $)^{(2)}$. The best known Lf property is its strong $\mathrm{Fe}$ binding ability. However, Lf function is not limited to $\mathrm{Fe}$ homoeostasis because Lf can display strong antimicrobial activity. Importantly, its localisation on the mucosal surface represents one of the first defence systems against microorganism invasion $^{(4)}$.

A growing body of evidence suggests that Lf plays a key role in host defence by modulating the innate and adaptive immune response ${ }^{(5)}$. The effects of $\mathrm{Lf}$ on the innate immune response are related in part to its ability to bind to conserved structures termed pathogen-associated molecular patterns (e.g. lipopolysaccharide
(LPS) on gram-negative bacteria and peptidoglycans on grampositive bacteria), that are critical for the activation of innate immunity. Lf is also capable of enhancing the activation of immune cells. Macrophages function as antigen-presenting cells (APC) to stimulate the development of antigen-specific $\mathrm{CD}^{+} \mathrm{T}$ cells, and Lf enhances their ability function as APC by stimulating the production of cytokines, such as IL-12, responsible for modulating development of Th1 cells ${ }^{(6,7)}$. In addition, human milk-derived Lf is observed to cause maturation of $\mathrm{CD}^{+} \mathrm{CD}^{+}$murine $\mathrm{T}$ cells, with a preference towards $\mathrm{CD} 4$ expression $^{(8)}$. In pigs, Lf decreases the recruitment of eosinophils to the duodenum through the intestinal Lf receptor $^{(9)}$. When administered orally, Lf has the ability to restore the host T-cell compartment, evident by an increase in splenic cellularity and enrichment of $\mathrm{CD}^{+} \mathrm{CD}^{+} \mathrm{T}$ cells ${ }^{(10)}$, suggesting that Lf may act as an immunostimulatory factor on the mucosal immune system.

Interestingly, Lf peptide fragments generated by proteolysis may be absorbed and capable of conserving the biological activity $^{(11)}$. Experiments have emphasised that a similar process

Abbreviations: Lf, lactoferrin: LPS, lipopolysaccharide: MPO, myeloperoxidase: NK, natural killer.

* Corresponding author: Y. Wang, fax +86 57188982650, email yzwang321@zju.edu.cn 
may participate in the bioactivity of orally administered $\mathrm{Lf}^{(12)}$. Indeed, recent experiments have suggested that these peptides play a beneficial role in microbial defence and cancer ${ }^{(13,14)}$. So far, the biological functions and mechanisms of action of these peptides are not fully understood.

LFP-20, an antimicrobial peptide located in the $\mathrm{N}$ terminus of porcine lactoferrin (pLf), displayed higher antimicrobial activity compared with their native protein counterpart ${ }^{(11,15,16)}$. LFP-20 exhibited anti-microbial effects on Escherichia coli, Staphylococcus aureus and Candida albicans ${ }^{(16)}$. Our previous study investigated that LFP-20 protects intestinal barrier through maintaining tight junction complex and modulating inflammatory response ${ }^{(17,18)}$. However, the mechanism of its antiinflammation action is in molecular or cellular layer is unknown. Here, an LPS-triggered systemic inflammatory response model was established. We elucidated the cellular mechanisms of the anti-inflammation effects of LFP-20, a peptide fragment of pLf, during LPS stimulation, thereby emphasising the importance of the LFP-20 in protecting from infection.

\section{Methods}

\section{Peptide synthesis}

LFP-20 (KCRQWQSKIRRTNPIFCIRR) was chemically synthesised by GL Biochem (Shanghai) Ltd, achieving 98\% purity of the synthetic peptide. The peptide was dissolved in endotoxinfree water and stored at $-80^{\circ} \mathrm{C}$.

\section{Animal model}

A total of seventy-two C57/BL6 male mice weighing 18-21 g (6-8 weeks) were obtained from Zhejiang Provincial Center for Disease Control and Prevention (Zhejiang CDC). Animals were individually housed and maintained on a $12 \mathrm{~h}$ light $-12 \mathrm{~h}$ dark cycle under specific pathogen-free conditions. All animals were provided with food and water ad libitum during the experimental period (1 week). Animal studies were approved by the Institutional Animal Care and Use Committee of Zhejiang University and performed in accordance with the institutional guidelines.

After 1 week of adaptation, the mice were randomly divided into six groups of twelve each: control, $5 \mathrm{mg} / \mathrm{kg}$ LFP-20 treatment; LPS treatment, $2.5 \mathrm{mg} / \mathrm{kg}$ LFP-20 pre-treatment followed by LPS treatment (LFPL + LPS); $5 \mathrm{mg} / \mathrm{kg}$ LFP-20 pre-treatment followed by LPS treatment (LFPM + LPS) and $10 \mathrm{mg} / \mathrm{kg}$ LFP-20 pre-treatment followed by LPS treatment (LFPH + LPS). The different concentrations of LFP-20 were injected intraperitoneally once daily for $6 \mathrm{~d}$, whereas the control and LPStreated groups were intra-peritoneally injected with an equal volume of sterile saline. On day 6 , mice in LPS group and LFP-20 (12.5, 25 and $50 \mu \mathrm{g} / \mathrm{ml})$ followed by LPS-treated groups were intra-peritoneally injected with LPS $(10 \mathrm{mg} / \mathrm{kg}$ mouse weight) $1 \mathrm{~h}$ after LFP-20 or saline treatment, and the other groups were injected with an equal volume of saline. All mice were euthanised by cervical dislocation $6 \mathrm{~h}$ after intraperitoneal injection of LPS or saline, and tissues and blood were collected ${ }^{(18)}$. The spleen and thymus were weighted as spleen or thymus index.

\section{Histopathology}

Intestinal tissues of the ileum were fixed in $4 \%$ paraformaldehyde solution immediately after the mice were euthanised. Tissues were embedded in paraffin and cut into 5-mm-thick sections. For the evaluation of histopathology changes, the tissues were stained with haematoxylin-eosin (H\&E) and observed under a microscope (Leica NEWDM 4500BR). Histopathological changes were graded on the histological injury scale described by Jang et al. ${ }^{(19)}$.

\section{Immunohistochemistry}

The distal ileum were fixed in $4 \%$ paraformaldehyde solution, embedded in paraffin and cut into slices longitudinally, subsequently permeabilised, stained and fixed. For the immunohistochemical analysis of CD68, anti-CD68 antibodies (Proteintech) were added at a dilution of 1:100 and incubated overnight at $4^{\circ} \mathrm{C}$ after blocking with $1 \%(\mathrm{w} / \mathrm{v})$ bovine serum albumin for $1 \mathrm{~h}$. After washing with PBS, samples were treated with horseradish peroxidase (HRP)-conjugated rabbit anti-goat IgG (HuaAn) at a ratio of $1: 100$. Samples were incubated at $4^{\circ} \mathrm{C}$ for $1 \mathrm{~h}$ and washed with PBS three times. 3,3'-Diaminobenzidine (DAB) (50-100 $\mu$; Dako) was added, and the slices were counterstained with haematoxylin. Finally, the samples were dehydrated in an ethanol (70-$100 \%$ gradient and treated with xylene to increase the transparency of slides. Neutral balsam was used for mounting.

Analysis of apoptotic death (terminal deoxyribonucleotidyl transferase-mediated biotin-16-dUTP nick-end labelling assay)

The cell apoptosis rate in the jejunum was detected using the In Situ Cell Death Detection Kit Fluorescein (Roche), according to the manufacturer's instructions. Proteinase $\mathrm{K}$ digestion was performed with $20 \mu \mathrm{g} / \mathrm{ml}$ Proteinase K (Qiagen). Healthy and apoptotic cells were counted in four to six randomly selected fields at $200 \times$ magnification. The percentage of apoptotic cells was taken as the percentage of the total number of terminal deoxyribonucleotidyl transferase-mediated biotin-16-dUTP nick-end labelling (TUNEL)-positive cells.

\section{Analysis of cytokines and myeloperoxidase in ileum}

Concentrations of several cytokines (interferon (IFN)- $\gamma$, TNF- $\alpha$, IL-6, IL-4, IL-5 and IL-12p70) were assessed using commercially available ELISA kits (R\&D Systems).

The activities of myeloperoxidase (MPO) were assessed by ELISA kit (Boster Wuhan), according to the manufacturer's instructions. Results were expressed in $\mathrm{U} /(\mathrm{mg}$ protein).

\section{Antibodies were determined by ELISA}

The concentration of antibodies was measured by ELISA kit. Antimouse IgA, IgM, IgE, IgG1, IgG2a, IgG2b and IgG3 antibody ELISA kits were purchased from eBiosciences. Plates were coated with $4 \mu \mathrm{g} / \mathrm{ml}$ anti-mouse IgA, IgM, IgE, IgG1, IgG2a, IgG2b or IgG3 antibodies overnight at $4{ }^{\circ} \mathrm{C}$. Then, diluted sera were added 
in triplicate to the plate for $1 \mathrm{~h}$ at $37^{\circ} \mathrm{C}$. Then after washing, $4 \mu \mathrm{g} / \mathrm{ml}$ HRP-conjugated anti-mouse IgA, IgM, IgE, IgG1, IgG2a, IgG2b or IgG3 antibodies were added to the plate and were incubated for another hour at $37^{\circ} \mathrm{C}$. Finally, the colour was developed by incubation with $o$-phenylenediamine. The optical density was read at $492 \mathrm{~nm}$ with an ELISA reader (Bio-Rad). Standard curves were established to quantitate the amounts of the respective cytokines.

\section{Flow cytometry-based immunophenotyping}

Cytometric analysis has been described in our previous studies ${ }^{(20-22)}$. Analysing the distribution of immune cell populations in lymphoid organs, single-cell suspensions were prepared from peripheral blood, mesenteric lymph nodes and spleen. Before analysis, cells were depleted from peripheral blood, mesenteric lymph nodes and spleen by ammonium chloride lysis. After blocking of non-specific binding using TruStain fcX ${ }^{\mathrm{TM}}$ (anti-mouse CD16/32) antibody, the immune cell population was stained with fluorochrome-labelled antibodies against phycoerythrin-Cy5 (PE-Cy5)anti-mouse CD3e, fluorescein isothiocyanate (FITC) anti-mouse CD4, phycoerythrin (PE) anti-mouse CD8a, FITC anti-mouse CD45R, and PE antimouse pan-natural killer (NK) cells. Isotype control antibodies (PE-Cy5-IgG2a, FITC-IgG2b and PE- IgG) were used as a negative

(A)

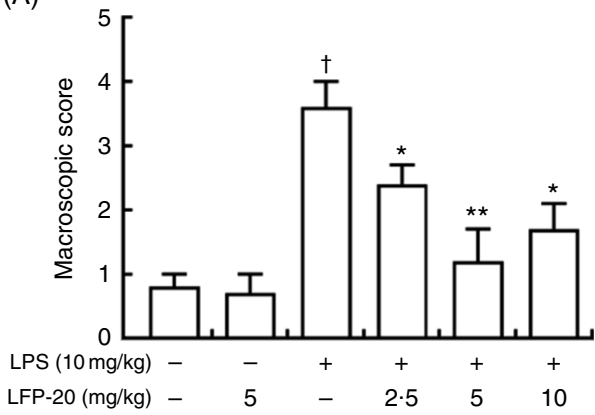

control. All antibodies were purchased from eBiosciences. Normalisation was done with respect to unstained cells. The samples were filtered immediately before analysis to remove any clumps. Data collection and analyses were performed on an FACS Calibur flow cytometer using CellQuest software.

\section{Statistical analysis}

Each experiment was performed on at least six animals per group. Statistical analyses were performed by one-way ANOVA, followed by the Duncan post hoc test with GraphPad Prism version 5.01 (GraphPad Software Inc.). Data are presented as means with their standard errors. An $\alpha$ value of $P<0.05$ was considered statistically significant.

\section{Results}

\section{Histopathological symptom analysis}

To assess the protection effects of LFP-20 on LPS-induced immune disorders, the distal ileum tissue was stained with H\&E. As shown in Fig. 1(A) and (C), histological examination of ileum tissue from the LPS-induced group revealed considerable tissue injury and immune disorder with extensive ulceration of the epithelial layer,
(B)

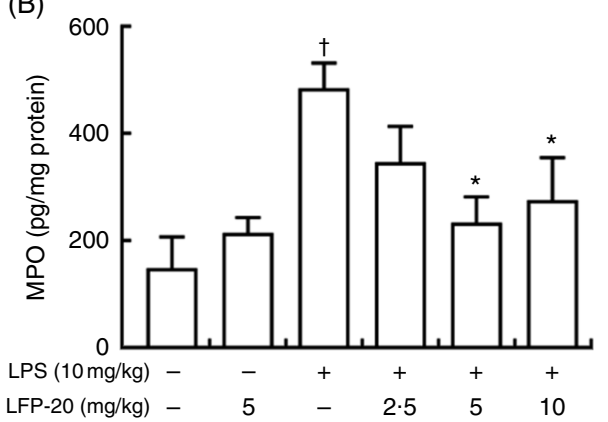

(C)
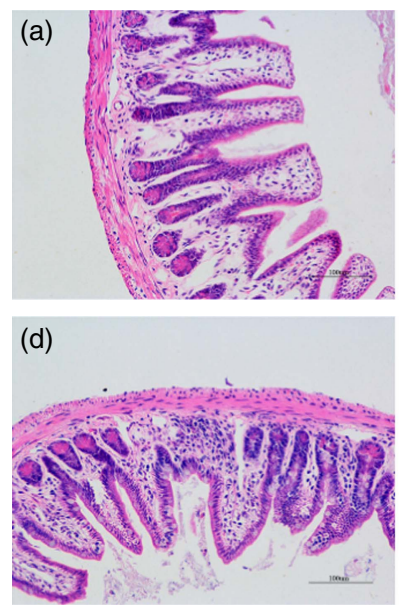
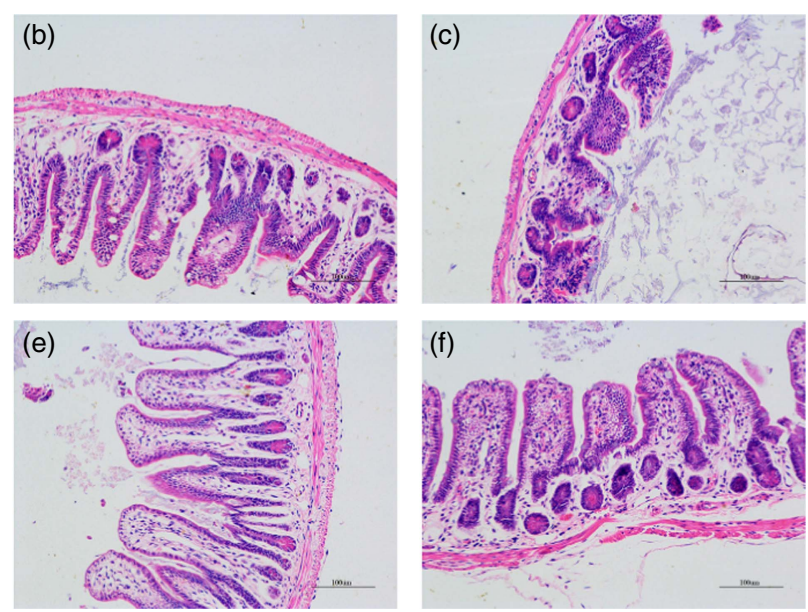

Fig. 1. Histopathological analysis of protection effects of LFP-20 on lipopolysaccharide (LPS)-induced lesions in ileum. (A) Levels of histopathological change quantified by mucosal damage grading. (B) Enzymatic activities of myeloperoxidase (MPO). (C) Representative haematoxylin-eosin-stained section from (C-a) control, (C-b) LFP-20, (C-c) LPS, (C-d) LFPL + LPS, (C-e) LFPM + LPS and (C-f) LFPH + LPS. Original magnification 200x. Values are means, with their standard errors represented by vertical bars. ${ }^{*} P<0.05$ compared with the LPS-stimulated group, $n 6$, biological replicates. $\dagger P<0.05$ compared with the respective control within columns. 
oedema, crypt damage to the bowel wall and infiltration of granulocytes and mononuclear cells into the mucosa. In contrast, LFP-20 pre-treatment reduced the LPS-induced symptom in ileum.

\section{Evaluation of macrophage and neutrophil infiltration in ileum mucosa}

MPO activity is an indicator for evaluating the degree of infiltration of neutrophils in tissues ${ }^{(23)}$. As shown in Fig. 1(B), MPO activity in ileum tissue from LPS-treated mice was markedly increased compared with control mice, whereas 5 or $10 \mathrm{mg} / \mathrm{kg}$ LFP-20 pre-treated mice showed significantly decreased MPO activities compared with the LPS-treated alone mice. Furthermore, the infiltration of CD68 cells into ileum tissue was detected by immunohistochemistry. In contrast to minimal infiltration of macrophages into the ileum of control mice, we observed increased infiltration of CD68 macrophages into the lesion area of ileum after LPS stimulation. Pre-treatment with LFP-20 reduced the infiltration of macrophages compared with the mice treated with LPS alone (Fig. 2). Together, LFP-20 pretreatment appeared to attenuate immune disorders in ileum during LPS stimulation.

\section{Level of apoptosis in intestinal tissue}

The TUNEL assay indicated that LPS treatment resulted in the apoptosis of epithelial cells in jejunum (brown signals, Fig. 3(C)), which was quantified by measuring the apoptosis index (Fig. 3(C)). Notably, compared with the apoptosis index of only the LPStreated group, pre-treatment of LPS-administered mice with LFP-20 significantly reduced the apoptosis index in a dose-dependent manner, though the apoptosis indexes in mice of LFP-20 group was similar to that in the control group. Meanwhile, the spleen and thymus are very important immune organs in body. The changes in thymus index and spleen index directly reflect the state of immune function. We found pre-treatment of LFP-20 significantly attenuated the LPS-increased spleen index, while the thymus index was not affected (Fig. 3(A) and (B)).

\section{Analysis of organ-specific immune cell subpopulation}

To address the hypothesis that the effect of LFP-20 on LPSinduced immune disorders in ileum was due to organ-specific immune homoeostasis, lymphoid and myeloid immune cell populations in peripheral blood, mesenteric lymph nodes and

(A)
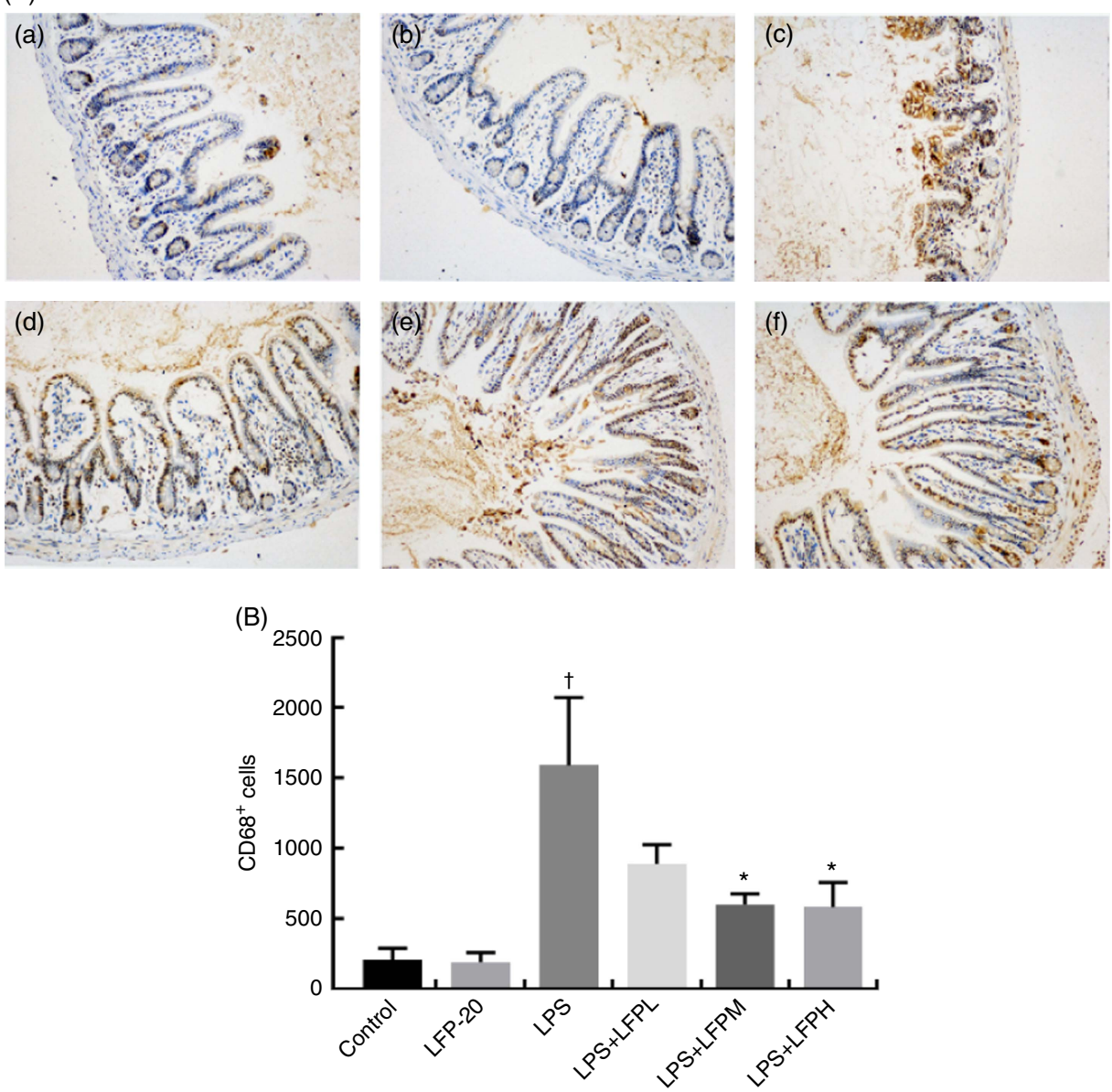

Fig. 2. Protective effects of LFP-20 on macrophage infiltration in ileum. (A) Infiltration of macrophage was represented by CD68 ${ }^{+}$cells. (a) Control, (b) LFP-20, (c) lipopolysaccharide (LPS), (d) LFPL + LPS, (e) LFPM + LPS and (f) LFPH + LPS. (B) The number of CD68 ${ }^{+}$cells counted according to the positive colour of brown and the average calculated. Original magnification 200x. Values are means, with their standard errors represented by vertical bars. ${ }^{\star} P<0.05$ compared with the LPS-stimulated group, $n 6$, biological replicates. $† P<0.05$ compared with the respective control within columns. 
(A)

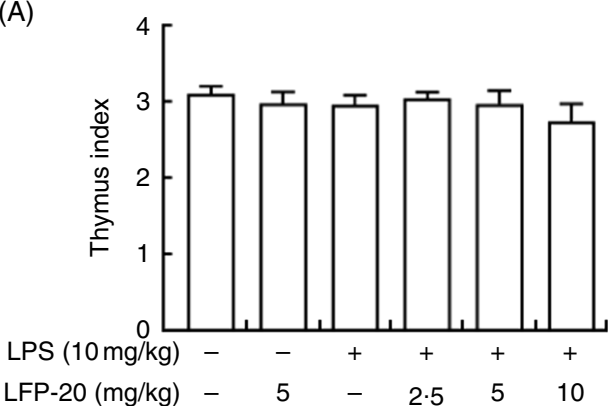

(B)

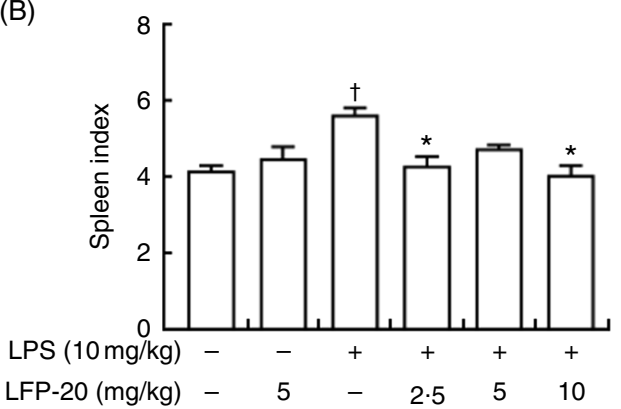

(C)
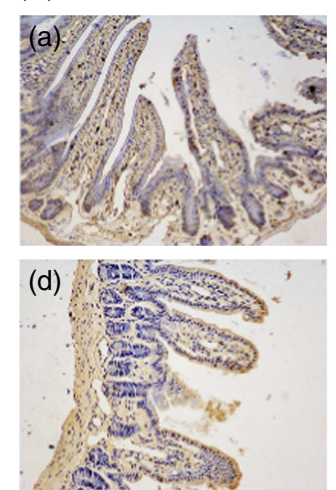
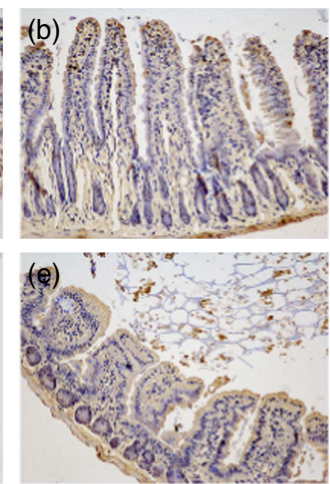
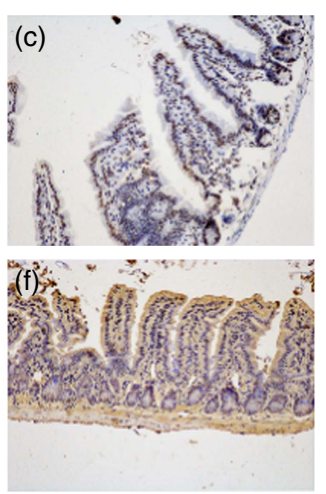

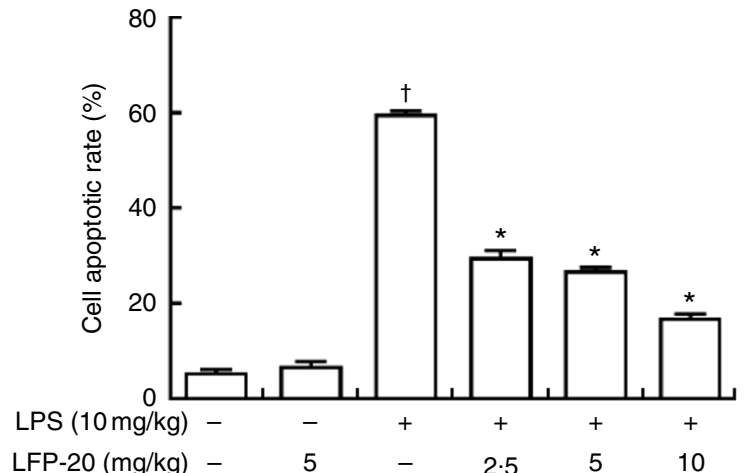

Fig. 3. Effect of LFP-20 on small-intestinal cell apoptosis and immune organ index. (A) Thymus index. (B) Spleen index. (C) Representative terminal deoxyribonucleotidyl transferase-mediated biotin-16-dUTP nick-end labelling images for cell apoptosis (brown signals, original magnification 200x) from (C-a) control, (C-b) LFP-20, (C-c) lipopolysaccharide (LPS), (C-d) LFPL + LPS, (C-e) LFPM + LPS, (C-f) LFPH + LPS. The right panel shows the apoptosis index of jejunal tissues. Values are means, with their standard errors represented by vertical bars. ${ }^{*} P<0.05$ compared with the LPS-stimulated group, $n 6$, biological replicates. $\dagger P<0.05$ compared with the respective control within columns.

Table 1. Effects of LFP-20 on immune cell subpopulations in peripheral blood (Mean values with their standard errors)

\begin{tabular}{|c|c|c|c|c|c|c|c|c|c|c|}
\hline \multirow[b]{2}{*}{ Group } & \multicolumn{2}{|c|}{$\mathrm{CD}^{+}{ }^{\mathrm{CD}} 4^{+}$} & \multicolumn{2}{|c|}{$\mathrm{CD}^{+} \mathrm{CD}^{+}$} & \multicolumn{2}{|c|}{$\mathrm{CD}^{+} \mathrm{CD}^{+} / \mathrm{CD}^{+} \mathrm{CD}^{+}$} & \multicolumn{2}{|c|}{ B cells } & \multicolumn{2}{|c|}{ NK cells } \\
\hline & Mean & SEM & Mean & SEM & Mean & SEM & Mean & SEM & Mean & SEM \\
\hline Control & $28 \cdot 38$ & 1.37 & 11.47 & 0.62 & 2.50 & 0.27 & 44.58 & 1.79 & 13.07 & 0.91 \\
\hline LFP-20 & $27 \cdot 86$ & 1.99 & 11.98 & 0.92 & $2 \cdot 37$ & 0.27 & $43 \cdot 10$ & 1.82 & $13 \cdot 83$ & 1.41 \\
\hline LPS & 32.40 & 1.35 & $7 \cdot 74^{\star}$ & 0.47 & $4 \cdot 18^{\star}$ & $0 \cdot 19$ & $52 \cdot 27^{\star}$ & 1.51 & $5 \cdot 64^{*}$ & 0.63 \\
\hline LFPL + LPS & $29 \cdot 83$ & 2.04 & 8.49 & 0.51 & 3.58 & $0 \cdot 26$ & $48 \cdot 18$ & 2.92 & $9.50 \dagger$ & $1 \cdot 17$ \\
\hline LFPM + LPS & $28 \cdot 74$ & 1.33 & $11.50 \dagger$ & 0.23 & $2.58 \dagger$ & 0.39 & $45 \cdot 04 \dagger$ & 1.53 & $12 \cdot 11 \dagger$ & 0.81 \\
\hline LFPH + LPS & $27 \cdot 60$ & 1.45 & $11.26 \dagger$ & 0.30 & $2.59 \dagger$ & $0 \cdot 17$ & $45 \cdot 59 \dagger$ & $2 \cdot 68$ & $11.69 \dagger$ & 1.09 \\
\hline
\end{tabular}

NK, natural killer; LPS, lipopolysaccharide.

${ }^{*} P<0.05$ compared with the respective control within columns.

$\dagger P<0.05$ compared with the LPS-stimulated group. $n 6$, biological replicates.

spleen were analysed. As shown in Table 1, compared with the control group, LPS stimulation markedly increased the percentages of $\mathrm{CD}^{+} \mathrm{CD}^{+} \mathrm{T}$ cells and $\mathrm{B}$ cells in the peripheral blood. Conversely, the percentages of $\mathrm{CD}^{+} \mathrm{CD}^{+} \mathrm{T}$ cells and NK cells in the peripheral blood were smaller in only the LPS-treated group compared with those in the control group. In contrast, LFP-20 pretreatment prevented the increase of $\mathrm{CD}^{+} \mathrm{CD} 4^{+} \mathrm{T}$ cells and $\mathrm{B}$ cells in peripheral blood during the LPS stimulation. As expected, the LPS-induced decreased percentages of $\mathrm{CD}^{+} \mathrm{CD} 8^{+} \mathrm{T}$ cells and NK cells in peripheral blood were also suppressed in LFP-20 pretreatment. Immune cell subpopulation in mice treated with
LFP-20 alone was similar to those of the control group. The similar effects were found in mesenteric lymph nodes and spleen, which further substantiated the immunomodulation effects of LFP-20 (Tables 2 and 3). Collectively, these results indicate that LFP-20 pre-treatment facilitated the balance of immune cells during LPS stimulation.

\section{Secretion of Th1- and Th2-cell-specific cytokines in ileum}

$\mathrm{T}$ lymphocytes mainly secrete cytokines and mediate inflammation-related immune responses ${ }^{(24)}$. To evaluate the 
Table 2. Effects of LFP-20 on immune cell subpopulations in spleen (Mean values with their standard errors)

\begin{tabular}{|c|c|c|c|c|c|c|c|c|c|c|}
\hline \multirow[b]{2}{*}{ Group } & \multicolumn{2}{|c|}{$\mathrm{CD}^{+} \mathrm{CD}^{+}$} & \multicolumn{2}{|c|}{$\mathrm{CD}^{+} \mathrm{CD}^{+}$} & \multicolumn{2}{|c|}{$\mathrm{CD}^{+} \mathrm{CD}^{+} / \mathrm{CD}^{+} \mathrm{CD}^{+}$} & \multicolumn{2}{|c|}{ B cells } & \multicolumn{2}{|c|}{ NK cells } \\
\hline & Mean & SEM & Mean & SEM & Mean & SEM & Mean & SEM & Mean & SEM \\
\hline Control & $27 \cdot 15$ & 1.17 & $11 \cdot 22$ & 0.78 & 2.46 & 0.26 & $45 \cdot 38$ & 2.05 & $13 \cdot 38$ & 1.14 \\
\hline LFP-20 & 31.24 & 0.59 & 11.39 & 0.59 & 2.75 & 0.10 & 47.68 & 3.43 & 14.20 & 1.18 \\
\hline LPS & $33.62^{*}$ & 1.22 & $8 \cdot 37^{*}$ & 0.20 & $4 \cdot 01^{*}$ & 0.07 & $51 \cdot 87^{*}$ & $1 \cdot 8$ & $6 \cdot 21^{*}$ & 0.78 \\
\hline LFPL + LPS & 29.34 & 1.35 & $11.09 \dagger$ & 0.08 & 2.65 & 0.12 & 47.65 & 3.22 & 6.84 & 1.07 \\
\hline LFPM + LPS & $28 \cdot 56$ & 1.28 & $12 \cdot 61 \dagger$ & 0.8 & $2 \cdot 30 \dagger$ & 0.25 & $48 \cdot 42$ & $2 \cdot 93$ & $11.75 \dagger$ & 1.07 \\
\hline LFPH + LPS & $27 \cdot 20 \dagger$ & 1.36 & $13 \cdot 28 \dagger$ & 0.44 & $2.04 \dagger$ & 0.45 & 49.02 & 3.88 & $15.40 \dagger$ & 1.46 \\
\hline
\end{tabular}

NK, natural killer; LPS, lipopolysaccharide.

${ }^{*} P<0.05$ compared with the respective control within columns.

$\dagger P<0.05$ compared with the LPS-stimulated group. $n 6$, biological replicates.

Table 3. Effects of LFP-20 on immune cell subpopulations in mesenteric lymph nodes (Mean values with their standard errors)

\begin{tabular}{|c|c|c|c|c|c|c|c|c|c|c|}
\hline \multirow[b]{2}{*}{ Group } & \multicolumn{2}{|c|}{$\mathrm{CD}^{+} \mathrm{CD}^{+}{ }^{+}$} & \multicolumn{2}{|c|}{$\mathrm{CD}^{+}{ }^{+} \mathrm{CD}^{+}{ }^{+}$} & \multicolumn{2}{|c|}{$\mathrm{CD}^{+} \mathrm{CD}^{+} / \mathrm{CD}^{+} \mathrm{CD}^{+}$} & \multicolumn{2}{|c|}{ B cells } & \multicolumn{2}{|c|}{ NK cells } \\
\hline & Mean & SEM & Mean & SEM & Mean & SEM & Mean & SEM & Mean & SEM \\
\hline Control & $20 \cdot 14$ & 1.01 & 11.51 & 1.3 & 1.89 & 0.24 & 24.39 & 2.05 & 7.60 & 0.83 \\
\hline LFP-20 & 21.59 & 1.46 & $12 \cdot 17$ & 0.58 & 1.71 & 0.16 & $25 \cdot 34$ & 0.61 & $9 \cdot 12$ & 0.61 \\
\hline LPS & 24.22 & 1.22 & $8 \cdot 33^{*}$ & 0.38 & $2 \cdot 90^{*}$ & 0.2 & $33.50^{*}$ & 1.42 & $3.40^{*}$ & 0.80 \\
\hline LFPL & 19.51 & 1.48 & 9.07 & 0.82 & $2 \cdot 27$ & 0.08 & 31.32 & 1.21 & 3.43 & 0.61 \\
\hline LFPM & $20 \cdot 61$ & $2 \cdot 11$ & $11.49 \dagger$ & 0.58 & $1.88 \dagger$ & 0.09 & 27.55 & 1.79 & 4.42 & 0.56 \\
\hline LFPH & 18.82 & 0.84 & $12 \cdot 61 \dagger$ & 1.69 & $1.62 \dagger$ & 0.22 & $26.65 \dagger$ & 0.88 & $6.42 \dagger$ & 0.82 \\
\hline
\end{tabular}

NK, natural killer; LPS, lipopolysaccharide.

* $P<0.05$ compared with the respective control within columns.

$\dagger P<0.05$ compared with the LPS-stimulated group. $n 6$, biological replicates.

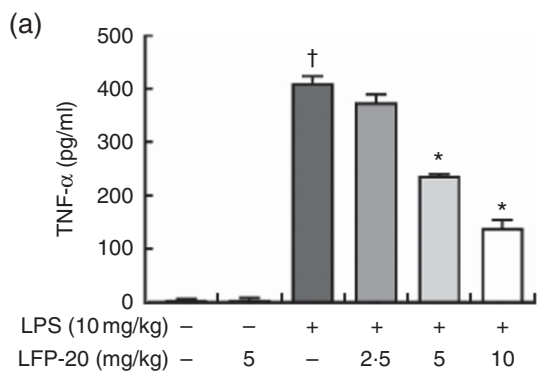

(d)

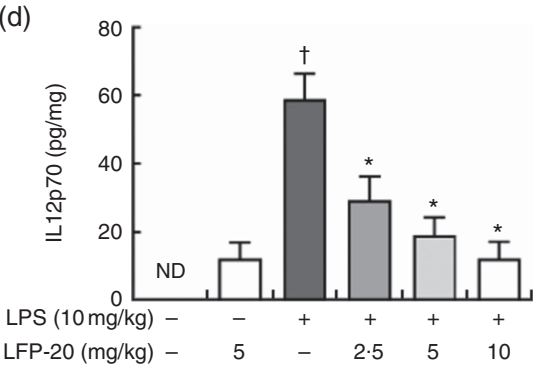

(b)

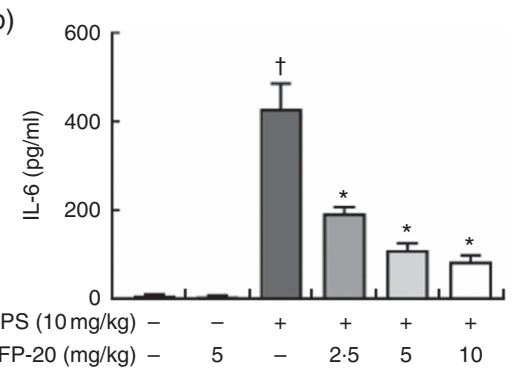

(e)

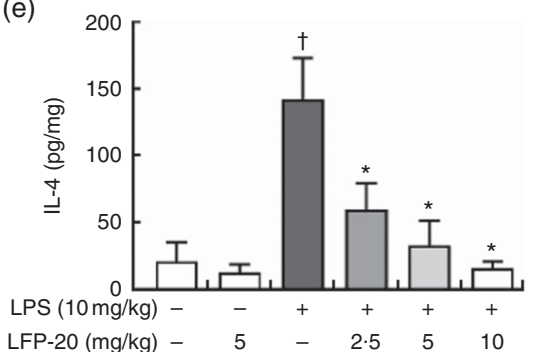

(c)

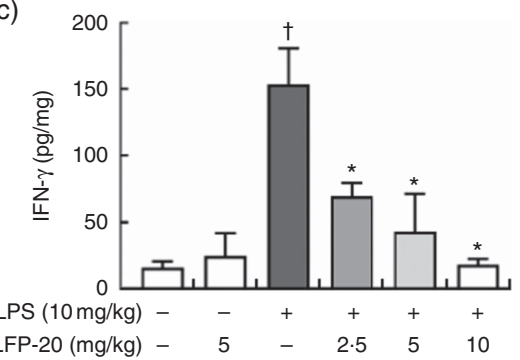

(f)

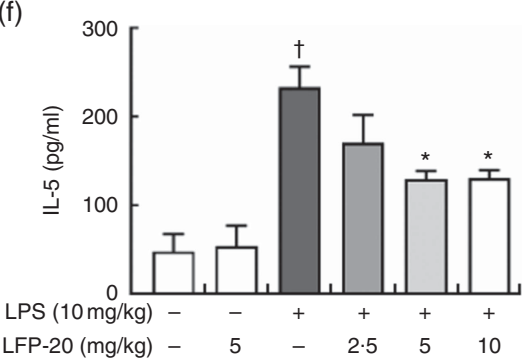

Fig. 4. Concentration of cytokines in ileum tissues. (a) TNF- $a$, (b) IL-6, (c) interferon (IFN)- $\gamma$, (d) IL-12p70, (e) IL-4 and (f) IL-5. Values are means, with their standard errors represented by vertical bars. ${ }^{*} P<0.05$ compared with the lipopolysaccharide (LPS)-stimulated group, $n 6$, biological replicates. $\dagger P<0.05$ compared with the respective control within columns. ND, not detected.

protective effects of LFP-20 against inflammation in LPS-stimulated mice, cytokines were detected by ELISA, including TNF- $\alpha$, IL-6, IFN- $\gamma$, IL-12p70, IL-4 and IL-5. As shown in Fig. 4(a)-(f), LPS treatment resulted in a significant increase in the concentration of TNF- $\alpha$, IL-6, IFN- $\gamma$, IL-12p70, IL- 4 and IL-5 in ileum, compared with untreated mice. Pre-treatment with LFP-20 markedly reduced LPSinduced cytokine secretion, which indicated that LFP-20 altered T lymphocyte functions and sustained a balanced Th1 and Th2 cell response. The concentration of cytokines in mice treated with LFP-20 alone was similar to the control group. 
(a)

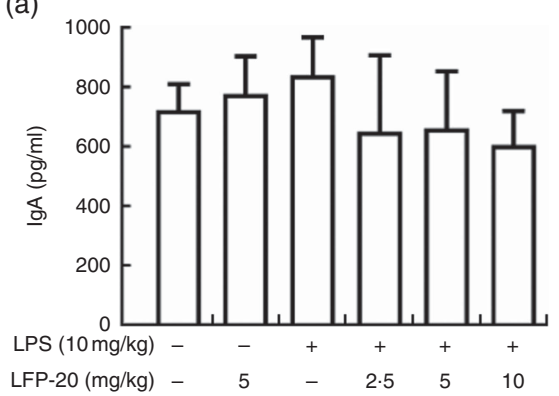

(b)

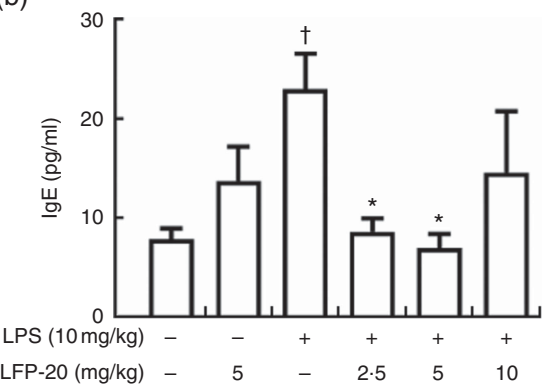

(c)

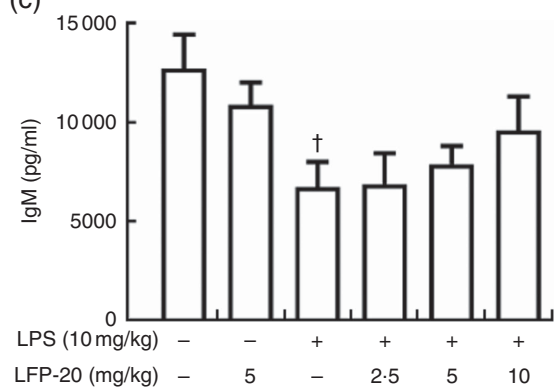

(d)

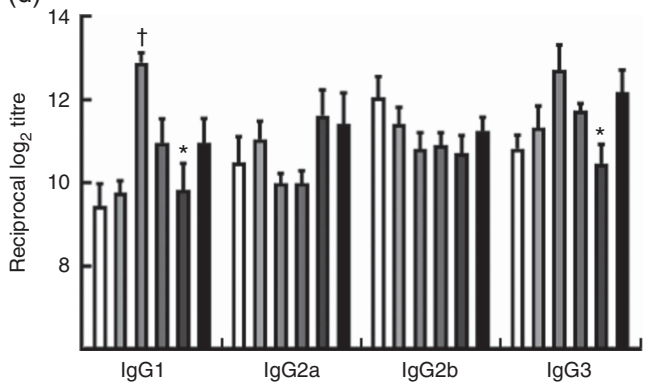

(e)

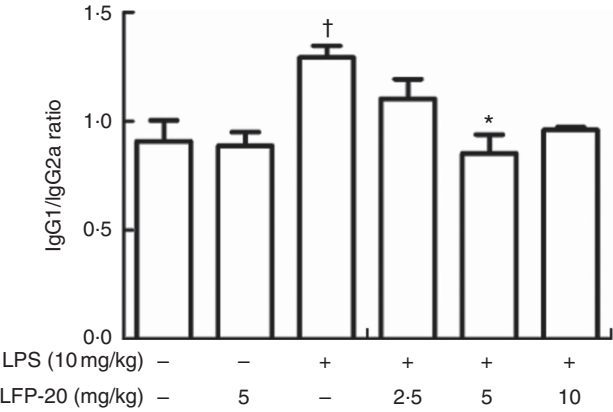

Fig. 5. Effects of LFP-20 on antibody production in lipopolysaccharide (LPS)-stimulated mice. (a) $\lg A$, (b) $\lg E$, (c) $\lg M$, (d) $\lg G$ subclasses and (e) the ratio of $\lg G 1 /$ IgG2a. Values are means, with their standard errors represented by vertical bars. ${ }^{*} P<0.05$ compared with the LPS-stimulated group, $n 6$, biological replicates. $\dagger P<0.05$ compared with the respective control within columns. $\square$, Control; $\square$, LFP-20; $\square$, LPS; $\square$, LPS + LFPL; $\square$, LPS + LFPM; $\square$, LPS + LFPH.

\section{Evaluation of antibody production in B cells after lipopolysaccharide stimulation}

Activation of B cells is usually accompanied by antibody production $^{(22)}$. We hypothesised that the LFP-20 sustained Th1 and Th2 response induced $\mathrm{B}$ cells to produce antibodies during LPS stimulation. To address this hypothesis, we investigated the level of antibody such as IgA, IgE, IgM, IgG1, IgG2a, IgG2b and IgG3. As shown in Fig. 5(a)-(d), during LPS stimulation, the level of IgM, IgA, IgG2a and IgG2b was not changed but the level of IgE, IgG1 and IgG3 increased. Pre-treatment with LFP-20 could decrease the elevated level of IgE, IgG1 and IgG3 dose dependently in LPS (Fig. 5(a)-(d)). Moreover, the increased ratio of IgG1/IgG2a in LPS, which represented Th2 response, was also blocked in LFP-20 pre-treated mice (Fig. 5(e)). The data demonstrated that LFP-20 regulated B-cell function through Th2 cell response.

\section{Discussion}

LFP-20, an Lf peptide fragment, is the nutritional active factor of Lf. Experiments have emphasised that this proteolysis generates peptide fragments capable of conserving the biological activity such as lactoferricin ${ }^{(11)}$. The model of LPS endotoxaemia mimics the endotoxin translocation from the intestine to blood circulation

This study assessed the protective effects of LFP-20 injected intra-peritoneally on inflammation response and intestinal lesions in LPS-stimulated mice. Further, by analysing the distribution and status of immune cells, we explored the cellular not molecular mechanisms.
The gut as a sensory organ could sense the invasion of pathogenic micro-organisms ${ }^{(25)}$. Intestinal mucosa is the first layer to defence against pathogens. Macrophages and dendritic cells are largely responsible for the innate immune response in the mucosa ${ }^{(26)}$. Consistent with the previous studies ${ }^{(27,28)}$, histopathological analysis revealed LPS stimulation marked atrophy and blunting of the villi, with discontinuous brush borders and disarrayed epithelium. Simultaneously, the infiltration of granulocytes and mononuclear cells into the mucosa was observed, which indicated that LPS stimulation triggered the innate immune response. Pre-treatment with $10 \mathrm{mg} / \mathrm{kg} \mathrm{LFP-20}$ reduced the histological evidence of LPS-induced symptom in ileum in a dose-dependent manner. MPO activity is directly proportional to neutrophil concentration in the inflamed tissue and is thus an index of neutrophil infiltration and inflammation $^{(29)}$. The results indicated that LPS-induced increased MPO activity was also significantly suppressed by LFP-20 pre-treatment. However, MPO is not produced exclusively by neutrophils and is also produced by monocytes and macrophage. In addition, the enzymatic activity of MPO can vary considerably between individuals even if the amount of MPO present is similar ${ }^{(30)}$. To substantiate this finding further, immunohistochemistry with CD68 antibody was conducted to assess infiltration of activated macrophages. As expected, pretreatment with LFP-20 restrained the infiltration of macrophage in ileum. Collectively, LFP-20 pre-treatment appears to attenuate exaggerated immune response triggered by LPS stimulation.

The observed basal differences in immune cell population located in non-lymphoid organs might result from an altered immune cell repertoire in lymphoid organs and/or immune cell traffic $^{(21)}$. In line with this thought, flow cytometry analysis of main immune cell subpopulation (T cells, B cells and NK cells) 
uncovered distinct changes in $\mathrm{CD}^{+} \mathrm{CD}^{+} \mathrm{T}$ cells, $\mathrm{CD}^{+} \mathrm{CD}^{+}$ $\mathrm{T}$ cells, B cells and NK cells counts in peripheral blood during LPS stimulation. In the presence of LFP-20, the mice revealed a reduced $\mathrm{CD}^{+} \mathrm{CD}^{+} \mathrm{T}$ cell and $\mathrm{B}$ cell population as well as an elevated amount of $\mathrm{CD}^{+} \mathrm{CD}^{+} \mathrm{T}$ cells and NK cells in the blood, as compared with LPS stimulation alone. To our surprise, the similar immunomodulation effects of LFP-20 on immune cell subpopulation were observed in mesenteric lymph nodes and spleen. This might reduce systemic inflammatory responses as these lymph nodes are drained by antigen presenting cells upon intra-peritoneal LPS treatment to promote the activation of adaptive immune responses by $\mathrm{T}$ and $\mathrm{B}$ cells.

Mechanistically, lymphocyte are immune effector cells, migrating from peripheral blood to tissues rapidly during infection via chemokine receptors and recognise the pathogens by pathogen recognition receptors ${ }^{(31)}$. T-cell-derived cytokines bound to B-cell cytokine receptors to promote B-cell proliferation, Ig class switching and somatic hypermutation as well as guide differentiation ${ }^{(32)}$. We found LFP-20 modulated the secretion of both activated Th1-cell-related IL-12p70, IFN- $\gamma$, TNF- $\alpha$ and Th2-cell-related IL-4, IL-5, IL-6, which suggested that LFP-20 contributed to a balanced Th1 and Th2 cell response. However, pre-treated with only LFP-20 suppressed LPS stimulation and up-regulated the secretion of antibody such as IgE, IgG1 and IgG3 but did not affect the level of IgM, IgA, IgG2a. Indeed, bovine and human Lf is capable of binding to surface receptors on the human T-cell line ${ }^{(33,34)}$. Lf can promote Th1 responses while inhibiting Th2 responses, and it causes T-cell receptor cross linking, which leads to the inhibition of T-cell activation, reduces the release of inflammatory factors such as IL-5 and IL-17 and further alleviates the degree of inflammation $^{(35)}$. Consistently, previous studies have shown that orally administered Lf has been demonstrated to increase the pool of $\mathrm{CD}^{+} \mathrm{T}$ cells, the secretion of IgA and IgG in murine mucosa with intestinal secretion ${ }^{(36,37)}$. These data suggested that Lf acted on $\mathrm{B}$ cells, which are well-known antigen presenters, to allow for their subsequent interaction with $\mathrm{T}$ cells, which favours elevation of the antibody response ${ }^{(36,38,39)}$. In addition, in a chemotherapy-induced immune suppression murine model, Lf administered intra-peritoneally was able to decrease the suppression of antibody-forming cells and facilitate the restoration of the immune response ${ }^{(40)}$. Collectively, our data demonstrated that LFP-20 regulated B-cell function through Th2 cell response. However, further study is needed to illustrate the signalling pathway of antibody secretion in cytokine-activated B cells.

Altogether, this study provided compelling evidence demonstrating that LFP-20 sustained immune homoeostasis through regulating cytokines and antibody secretion to defend LPS-triggered systemic inflammatory response. The insights obtained from this study further advance our understanding of the nutritional value of Lf and highlight the importance of its peptide in the development of immune response.

\section{Acknowledgements}

The authors would like to thank Wang lab members for helpful discussion.
This study was funded by a grant from the National Natural Science Foundation of China, number 3163000269 and a grant from the Modern Agroindustry Technology Research System, number CARS-36.

$\mathrm{X}$. Z. and Y. W conceived the project, designed the experiments. X. Z., H. W. and J. Z. performed the experiments. X. Z wrote the manuscripts. All authors discussed the results and edited the manuscript.

Authors declare that they have no conflict of interest in relation to this study.

\section{References}

1. Lambert LA, Perri H \& Meehan TJ (2005) Evolution of duplications in the transferrin family of proteins. Comp Biochem Physiol B Biochem Mol Biol 140, 11-25.

2. Liao YL, Alvarado R, Phinney B, et al. (2011) Proteomic characterization of human milk whey proteins during a twelve-month lactation period. J Proteome Res 10, 1746-1754.

3. Rosa L, Cutone A, Lepanto MS, et al. (2017) Lactoferrin: a natural glycoprotein involved in iron and inflammatory homeostasis. Int J Mol Sci 18, 1985.

4. Jenssen H \& Hancock REW (2009) Antimicrobial properties of lactoferrin. Biochimie 91, 19-29.

5. Telang S (2018) Lactoferrin: a critical player in neonatal host defense. Nutrients 10, 1228.

6. Actor JK, Hwang SA \& Kruzel ML (2009) Lactoferrin as a natural immune modulator. Curr Pharm Design 15, 1956-1973.

7. Trinchieri G (2003) Interleukin-12 and the regulation of innate resistance and adaptive immunity. Nat Rev Immunol 3, 133-146.

8. Zimecki M, Mazurier J, Machnicki M, et al. (1991) Immunostimulatory activity of lactotransferrin and maturation of $\mathrm{CD}^{-}$ $\mathrm{CD}^{-}$murine thymocytes. Immunol Lett 30, 119-124.

9. Cooper C, Nonnecke E, Lonnerdal B, et al. (2014) The lactoferrin receptor may mediate the reduction of eosinophils in the duodenum of pigs consuming milk containing recombinant human lactoferrin. Biometals 27, 1031-1038.

10. Artym J, Zimecki M \& Kruzel ML (2003) Reconstitution of the cellular immune response by lactoferrin in cyclophosphamidetreated mice is correlated with renewal of $\mathrm{T}$ cell compartment. Immunobiology 207, 197-205.

11. Bellamy W, Takase M, Wakabayashi H, et al. (1992) Antibacterial spectrum of lactoferricin $\mathrm{B}$, a potent bactericidal peptide derived from the N-terminal region of bovine lactoferrin. J Appl Bacteriol 73, 472-479.

12. Dallas DC, Guerrero A, Khaldi N, et al. (2014) A peptidomic analysis of human milk digestion in the infant stomach reveals protein-specific degradation patterns. J Nutr 144, 815-820.

13. Yin C, Wong JH \& Ng TB (2014) Recent studies on the antimicrobial peptides lactoferricin and lactoferrampin. Curr Mol Med 14, 1139-1154.

14. Yin CM, Wong JH, Xia J, et al. (2013) Studies on anticancer activities of lactoferrin and lactoferricin. Curr Protein Pept Sci 14, 492-503.

15. Bellamy W, Wakabayashi H, Takase M, et al. (1993) Killing of Candida albicans by lactoferricin B, a potent antimicrobial peptide derived from the N-terminal region of bovine lactoferrin. Med Microbiol Immunol 182, 97-105.

16. Han FF, Gao YH, Luan C, et al. (2013) Comparing bacterial membrane interactions and antimicrobial activity of porcine lactoferricin-derived peptides. J Dairy Sci 96, 3471-3487.

17. Zong X, Hu WY, Song DG, et al. (2016) Porcine lactoferrinderived peptide LFP-20 protects intestinal barrier by 
maintaining tight junction complex and modulating inflammatory response. Biochem Pharmacol 104, 74-82.

18. Zong X, Song D, Wang T, et al. (2015) LFP-20, a porcine lactoferrin peptide, ameliorates LPS-induced inflammation via the MyD88/NF-kappa B and MyD88/MAPK signaling pathways. Dev Comp Immunol 52, 123-131.

19. Jang S-E, Hyam SR, Jeong J-J, et al. (2013) Penta-O-galloylbeta-D-glucose ameliorates inflammation by inhibiting MyD88/NF-kappa B and MyD88/MAPK signalling pathways. Br J Pharmacol 170, 1078-1091.

20. Kong B, Wang X, Yang W, et al. (2018) Effects of simvastatin on the function of splenic $\mathrm{CD}^{+}$and $\mathrm{CD}^{+} \mathrm{T}$ cells in sepsis mice. Immunol Res 66, 355-366.

21. Pohlmann S, Scheu S, Ziegler V, et al. (2018) Hepatic Rac1 GTPase contributes to liver-mediated basal immune homeostasis and LPS-induced endotoxemia. Biochim Biophys Acta 1865, 1277-1292.

22. Zhu GZ, Wang XQ, Xiao H, et al. (2017) Both Notch1 and its ligands in B cells promote antibody production. Mol Immunol 91, $17-23$.

23. Zhang H, Xia X, Han F, et al. (2015) Cathelicidin-BF, a novel antimicrobial peptide from Bungarus fasciatus, attenuates disease in a dextran sulfate sodium model of colitis. Mol Pharm 12, 1648-1661.

24. Lythe G \& Molina-Paris C (2018) Some deterministic and stochastic mathematical models of naive T-cell homeostasis. Immunol Rev 285, 206-217.

25. Furness JB, Rivera LR, Cho H-J, et al. (2013) The gut as a sensory organ. Nat Rev Gastroenterol Hepatol 10, 729-740.

26. Santaolalla R \& Abreu MT (2012) Innate immunity in the small intestine. Curr Opin Gastroenterol 28, 124-129.

27. Song DG, Zong X, Zhang HW, et al. (2015) Antimicrobial peptide cathelicidin-BF prevents intestinal barrier dysfunction in a mouse model of endotoxemia. Int Immunopharmacol 25, 141-147.

28. Han F, Zhang H, Xia X, et al. (2015) Porcine beta-defensin 2 attenuates inflammation and mucosal lesions in dextran sodium sulfate-induced colitis. J Immunol 194, 1882-1893.

29. Choudhary S, Keshavarzian A, Yong S, et al. (2001) Novel antioxidants zolimid and AEOL11201 ameliorate colitis in rats. Dig Dis Sci 46, 2222-2230.
30. Bustos D, Greco G, Yapur V, et al. (2000) Quantification of fecal neutrophils by MPO determination (myeloperoxidase) in patients with invasive diarrhea [Cuantificacion de neutrofilos fecales mediante la determinacion de MPO (Mieloperoxidasa) en pacientes con diarrea invasiva]. Acta Gastroenterol Latinoam 30, 85-87.

31. Zhao Y, Zou W, Du J, et al. (2018) The origins and homeostasis of monocytes and tissue-resident macrophages in physiological situation. I Cell Physiol 233, 6425-6439.

32. Crotty S (2015) A brief history of T cell help to B cells. Nat Rev Immunol 15, 185-189.

33. Legrand D, van Berkel PHC, Salmon V, et al. (1997) The N-terminal $\operatorname{Arg}(2), \operatorname{Arg}(3)$ and $\operatorname{Arg}(4)$ of human lactoferrin interact with sulphated molecules but not with the receptor present on Jurkat human lymphoblastic T-cells. Biochem J 327, 841-846.

34. Manzoni P, Stolfi I, Messner H, et al. (2012) Bovine lactoferrin prevents invasive fungal infections in very low birth weight infants: a randomized controlled trial. Pediatrics 129, 116-123.

35. Wang SB, Deng YQ \& Ren J (2013) Lactoferrin administration into the nostril alleviates murine allergic rhinitis and its mechanisms. Scand J Immunol 78, 507-515.

36. Sfeir RM, Dubarry M, Boyaka PN, et al. (2004) The mode of oral bovine lactoferrin administration influences mucosal and systemic immune responses in mice. J Nutr 134, 403-409.

37. Manzoni P, Meyer M, Stolfi I, et al. (2014) Bovine lactoferrin supplementation for prevention of necrotizing enterocolitis in very-low-birth-weight neonates: a randomized clinical trial. Early Hum Dev 90, Suppl. 1, S60-S65.

38. Siqueiros-Cendon T, Arevalo-Gallegos S, Iglesias-Figueroa BF, et al. (2014) Immunomodulatory effects of lactoferrin. Acta Pharmacol Sin 35, 557-566.

39. Debbabi H, Dubarry M, Rautureau M, et al. (1998) Bovine lactoferrin induces both mucosal and systemic immune response in mice. J Dairy Res $\mathbf{6 5}, 283-293$.

40. Artym J, Zimecki M, Kuryszko J, et al. (2005) Lactoferrin accelerates reconstitution of the humoral and cellular immune response during chemotherapy-induced immunosuppression and bone marrow transplant in mice. Stem Cells Dev 14, $548-555$. 